\title{
Adaptación de la subescala de marihuana de la escala de Autoeficacia para el Rechazo del Consumo de Drogas (DURSE) en adolescentes
}

\section{Adaptation of the Marihuana Subscale of the Drug Use Resistance Self-Efficacy (DURSE) Scale in Adolescents}

\author{
David Pascual Olivo Chang ${ }^{\mathrm{a}, *}$, Cecilia Chau Pérez-Aranibar ${ }^{\mathrm{a}}$, \\ Fiorella Otiniano Campos ${ }^{\mathrm{a}}$, Silvana Melissa Romero Saletti ${ }^{\mathrm{a}}$

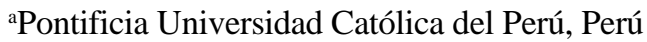

\section{Resumen}

Antecedentes: la autoeficacia es un factor relevante en el consumo de drogas y existe una necesidad de contar con instrumentos de medición para la investigación adaptados en el Perú. La escala de Autoeficacia para el Rechazo del Consumo de Drogas (DURSE, por sus siglas en inglés; Carpenter, 2006) es un instrumento útil para medir este aspecto en diferentes situaciones sociales. Objetivo: evaluar las propiedades psicométricas de validez y confiabilidad de la subescala de marihuana de la escala DURSE en escolares de colegios nacionales de Lima. Método: se tradujo la escala y se sometió a un piloto con 83 adolescentes. La versión final fue aplicada a una muestra de 1015 estudiantes de secundaria. Resultados: el análisis factorial confirmatorio demostró una estructura unifactorial con buen ajuste (CFI = .990; TLI = .986; RMSEA $=.057 ;$ SRMR $=.015)$ y se obtuvo una alta confiabilidad (.98). Se analizó la invarianza según sexo del participante, encontrándose invarianza escalar, pero no métrica. Se obtuvieron mayores niveles de autoeficacia en quienes no habían consumido marihuana, no tenían intención de probar, y rechazaron ofertas de consumo. Conclusiones: la subescala de marihuana de la escala DURSE es un instrumento idóneo para medir la autoeficacia para el rechazo de consumo de marihuana en adolescentes de Lima.

Palabras clave: autoeficacia; drogas; marihuana; adolescentes.

Para citar este artículo:

Olivo, D. P., Chau, C., Otiniano, F., \& Romero, S. M. (2021). Adaptación de la subescala de marihuana de la escala de Autoeficacia para el Rechazo del Consumo de Drogas (DURSE) en adolescentes. Liberabit, 27(2), e434. https://doi.org/10.24265/ liberabit.2021.v27n2.02

* david.olivo.chang@gmail.com

\begin{abstract}
Background: self-efficacy is an important factor in drug use and there is a need for adapting psychological measuring instruments to the Peruvian context. The Drug Use Resistance Self-Efficacy (DURSE) scale (Carpenter, 2006) is a useful instrument for measuring this construct across different social situations. Objectives: to assess the psychometric properties of validity and reliability of the DURSE marijuana subscale among public school students from Lima. Method: the scale was translated and subjected to a pilot study consisting of 83 adolescents. The final version was administered to a sample of 1015 high school students. Results: the confirmatory factor analysis showed a one-factor structure with good fit indices $(\mathrm{CFI}=.990, \mathrm{TLI}=.986, \mathrm{RMSEA}=.057, \mathrm{SRMR}=.015)$ and a high reliability coefficient (.98). The invariance was analyzed considering the participants' sex, thus finding scalar but not metric invariance. Higher levels of selfefficacy were found among students who had not used marijuana, had no intention of trying it and refused marijuana offers. Conclusions: the DURSE marijuana subscale is a suitable instrument to measure marijuana use resistance self-efficacy among adolescents from Lima.
\end{abstract}

Keywords: self-efficacy; drugs; marijuana; adolescents.

Este es un artículo Open Access publicado bajo la licencia Creative Commons Atribución 4.0 Internacional. (CC-BY 4.0)

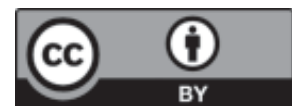

Universidad de San Martín de Porres, Lima - Perú http://ojs3.revistaliberabit.com 


\section{Introducción}

El consumo de marihuana se ha incrementado en los últimos años, especialmente, en los lugares que la han legalizado. Asimismo, el número de personas que requiere tratamiento por desórdenes de uso de drogas, cuya droga principal es la marihuana, ha aumentado en todas las regiones, excepto África (Naciones Unidas, 2019). Por otro lado, la potencia de esta droga, que depende de mayores concentraciones del componente psicoactivo de la marihuana denominado Tetrahidrocannabinol (THC), se ha incrementado también (Centro de Información y Educación para la Prevención del Abuso de Drogas [CEDRO], 2018; Naciones Unidas, 2019).

Esta sustancia es consumida principalmente en poblaciones adolescentes escolares o universitarias (Organización de los Estados Americanos [OEA], 2019). En América Latina, los niveles de prevalencia son muy heterogéneos y en la mayoría de los países, se muestra una tendencia a su aumento en la población general, así como en la población escolar de secundaria (OEA, 2019).

De acuerdo con las encuestas de CEDRO (2017), en el Perú, el consumo de marihuana presenta una prevalencia de vida general de $8.1 \%$, en el caso de adolescentes entre 12 y 18 años es de $2.7 \%$, habiendo importantes diferencias por sexo, ya que es mayor en hombres (15.5\%) que en mujeres (2.6\%). Asimismo, en alumnos de secundaria, cabe resaltar que, si bien Perú presenta tasas más bajas en relación a otros países, es posible que esta población sea más vulnerable a sus efectos negativos, ya que el porcentaje de consumidores problemáticos representa prácticamente la mitad de aquellos que consumieron el año anterior -y la cuarta parte de aquellos que consumieron alguna vez en su vida-. De aquellos con consumo problemático, la mitad tiene un grado de riesgo alto (Comisión Nacional para el Desarrollo y Vida sin Drogas [DEVIDA], 2014a).

Una variable asociada al inicio y mantenimiento del consumo de marihuana es la autoeficacia para resistirse al consumo de drogas. Esta puede definirse como la habilidad percibida para rechazar exitosamente las ofertas de consumir drogas en diferentes situaciones de presión (Carpenter, 2006). Esta habilidad se enmarca dentro de la teoría cognitiva social según la cual el comportamiento es determinado por la interacción de factores ambientales, personales y conductuales (Bandura, 1986). Entre los factores personales son de particular importancia las expectativas de resultado y eficacia, las cuales se construyen a partir de la observación de relaciones condicionales entre eventos y los resultados que producen las acciones. De esta manera, las creencias acerca de la propia eficacia se encuentran a la base de la agencia humana, ya que es necesario el incentivo de creer en la capacidad de lograr resultados deseados o prevenir resultados perjudiciales para actuar o perseverar ante las dificultades (Bandura, 2001).

En ese sentido, se ha encontrado que la autoeficacia respecto al consumo de marihuana es un importante factor protector para el consumo en adolescentes y está relacionada a su vez con otro factor de riesgo crucial que es el consumo de pares (U.S. Department of Health and Human Services, 2016).

Asimismo, diversas investigaciones encuentran que la autoeficacia respecto al consumo está asociada con la intención de consumir y al consumo en sí mismo (D’Amico et al., 2020; Lopez-Quintero \& Neumark, 2015; Morell-Gomis et al., 2019), y puede mediar entre la impulsividad y el uso de marihuana (Papinczak et al., 2018). Además, está asociada a una menor percepción de daño por su uso (Zullig \& Valois, 2017). Finalmente, entre consumidores usuales se encuentra que una mayor autoeficacia está relacionada al uso de estrategias protectoras para reducir los daños derivados del consumo (Pearson et al., 2019).

En relación con el aspecto del tratamiento, se encuentra que es un componente importante en las intervenciones grupales para reducir las ansias de 
consumir en personas con abuso de drogas (Sheykhnezhad \& Seyedfatemi, 2019), en las primeras sesiones de tratamiento con adolescentes (Chung \& Maisto, 2016), y en las barreras percibidas para dejar la marihuana (Zvolensky et al., 2018). Asimismo, está asociada a lapsos de recaída en procesos de abstinencia (Shrier et al., 2018), así como a una menor probabilidad de abstinencia en tratamientos para la dependencia, especialmente en lo referente a autoeficacia para abstenerse de consumir en situaciones de alto afecto negativo (Gullo et al., 2017).

En el contexto nacional y local, un estudio en Lima-Perú encontró que un $7 \%$ de adolescentes de $4 .^{\circ}$ y $5 .^{\circ}$ de secundaria reportan que sí consumirían una droga si un amigo les invitara, y un 15\% tal vez lo haría (Zavaleta et al., 2006).

Por los motivos anteriormente mencionados, el desarrollo de la autoeficacia para resistirse a las ofertas de consumo de drogas es un componente importante de los programas de prevención tanto en el Perú (DEVIDA, 2014b) como en programas exitosos basados en evidencia en diversos países a nivel escolar, tales como Life Skills Training (National Health Promotion Associates, 2017), keepin' it Real (Real Prevention, 2019), y el Proyecto para el No Abuso de Drogas (University of Southern California Institute for Prevention Research, 2019); así como también de intervenciones breves vía computadora, como Project Chill (Walton et al., 2013).

En la actualidad, existen pocos instrumentos que midan adecuadamente la autoeficacia para rechazar el consumo de drogas en adolescentes (Carpenter \& Howard, 2009). Entre los más usados se puede encontrar el Drug-Taking Confidence Questionnaire (DTCQ; Sklar et al., 1997) que fue desarrollado en pacientes que se encuentran buscando tratamiento y está destinado a adultos. Cuenta con diversas preguntas que miden la autoeficacia en situaciones de emociones desagradables, incomodidad física, emociones agradables, prueba del control personal, ansias y tentación de consumir, conflicto con otros, presión social, y momentos placenteros con otros. Este instrumento cuenta con una versión breve validada en adolescentes en tratamiento (Ramo et al., 2009). Por otro lado, se encuentra el Drug Avoidance SelfEfficacy Scale (DASES; Martin et al., 1995), desarrollado en jóvenes usuarios de drogas, cuyos ítems describen distintas situaciones referentes a estados emocionales negativos, positivos y algunas situaciones sociales, principalmente orientado a personas que han consumido previamente o que estén en tratamiento. Por último, el Cannabis Refusal Self Efficacy Questionnaire (CRSEQ; Young et al., 2012) desarrollado en adultos referidos para evaluación por uso de marihuana, que mide aspectos de alivio emocional, rechazo de oportunidades y facilitación social.

Se puede observar que los instrumentos disponibles están frecuentemente orientados a adultos y en varios casos a adolescentes consumidores, siendo investigados en poblaciones en tratamiento. Asimismo, hacen referencia a diversos aspectos de la autoeficacia, tales como las ansias de consumir para aliviar estados emocionales negativos o exacerbar estados positivos, pero dejando de lado las diversas dificultades inherentes a distintas situaciones sociales en las que los adolescentes se ven sujetos a ofertas de consumo. Asimismo, solo dos de estas escalas tienen adaptaciones al idioma español con muestras pequeñas. Por ejemplo, de las escalas mencionadas anteriormente, el DTCQ fue adaptado y validado en Perú en una muestra de 154 pacientes varones con adicciones (Vigo, 2003); mientras que el DASES fue adaptado y validado en Argentina en una muestra de 70 hombres y mujeres mayores de 16 años internados o en tratamiento ambulatorio por conductas adictivas (Etchemendi et al., 2019).

Sin embargo, es evidente la relevancia de medir específicamente la autoeficacia para el rechazo de consumo de marihuana, dada su importancia como factor protector y factor de riesgo del consumo de 
pares en la adolescencia; mas no se cuenta con un instrumento breve, enfocado en adolescentes, que apoye a la prevención a temprana a edad, por lo expuesto anteriormente. Adicionalmente, es importante que los ítems permitan cubrir diversas situaciones sociales en las que el adolescente puede verse expuesto a ofertas de consumo de drogas, aspecto en el cual las escalas existentes son limitadas (Carpenter, 2006).

Una escala específica para el rechazo de consumo que resulta particularmente útil en este sentido es la escala de Autoeficacia para el Rechazo de Consumo de Drogas (DURSE por sus siglas en inglés; Carpenter, 2006; Carpenter \& Howard, 2009). Esta fue desarrollada con escolares de Estados Unidos y construida guardando estrecha relación con los lineamientos de Bandura para la construcción de escalas de autoeficacia, haciendo preguntas sobre juicios probabilísticos de capacidades formuladas en tiempo presente (Bandura, 2001; Carpenter, 2006). Asimismo, esta escala fue creada para medir la autoeficacia para el rechazo de consumo de tres sustancias: alcohol, marihuana y tabaco. Cada una posee 8 ítems que presentan diversas situaciones en las que podrían ofrecerse las sustancias. Cabe resaltar que los ítems para las tres sustancias son los mismos y están redactados de la misma manera. Sin embargo, lo que cambia es la sustancia ofrecida.

En su primera validación, se hallaron valores de confiabilidad mediante el coeficiente alpha de Cronbach de .98, .95 y .97 para las subescalas de marihuana, alcohol y tabaco, respectivamente. Asimismo, se halló una adecuada validez factorial mediante un análisis exploratorio (usando el método de extracción de componentes principales con rotación Varimax) con un modelo de 3 factores que explicó el 83.09\% de la varianza. El factor de marihuana explicó el 34.14\% de la varianza; el factor de tabaco, el $24.29 \%$ de la varianza; y el factor de alcohol, el $24.17 \%$ de la varianza en una muestra de 223 adolescentes en su mayoría de 12 años de Estados Unidos (Carpenter \& Howard, 2009).
Asimismo, la escala DURSE ha mostrado correlacionar negativamente con la intención de uso de drogas, así como ha mostrado evidencias de validez convergente con la Wake Forest Drug Refusal Skills Scale $(r=.48)$ y la Botvin Refusal Skills Scale $(r=.40)$ (Carpenter \& Howard, 2009).

Con relación a diferencias de sexo, si bien se ha encontrado que la escala DURSE no muestra diferencias significativas entre hombres y mujeres, $\mathrm{y}$ que su estructura factorial en un análisis exploratorio resultó ser bastante similar entre ambos (Carpenter, 2006), las autoras indagaron sobre este aspecto dado que puede existir diferencias debido a las distintas expectativas, creencias y preocupaciones que tienen hombres y mujeres sobre situaciones de oferta de drogas. Al desarrollar el instrumento, encontraron que las mujeres estaban más preocupadas por que les ofrezcan drogas en fiestas y que las ofertas más realistas eran las realizadas por una pareja (Carpenter, 2006). Otras validaciones de instrumentos del mismo constructo han indagado a su vez en diferencias por sexo, pero no las han encontrado (Choi et al., 2013; Young et al., 2012).

Por otro lado, puntajes altos en la escala DURSE se vieron asociados a una baja intención de consumir alcohol, marihuana o tabaco en los siguientes 12 meses $(r=-.24)$. Sin embargo, la escala DURSE ha mostrado una pequeña asociación positiva con la deseabilidad social ( $r=.15$ ), siendo probable que los adolescentes crean que rechazar ofertas para consumir una droga sea una conducta socialmente deseable. Este y otros sesgos se pueden reflejar en la distribución asimétrica de los puntajes de la escala, lo cual también puede dar cuenta que los adolescentes sobreestiman su juicio sobre su autoeficacia debido a inexperiencia o poca familiaridad (Carpenter \& Howard, 2009).

El presente estudio se enmarca en el contexto de otra investigación de mayor alcance sobre los factores de riesgo para el consumo de marihuana en escolares, dentro de los cuales la autoeficacia es 
considerada. Por ello, el interés de presentar dichos hallazgos aquí es el de proveer de una adaptación de la subescala de marihuana de la escala DURSE. En el contexto de dicha investigación y considerando que una batería de pruebas muy extensa podría incidir en la atención de los adolescentes y, por lo tanto, en su respuesta, así como sobrepasar el tiempo brindado por los colegios, se optó por solo aplicar la subescala de marihuana de la escala DURSE. Cabe resaltar que, si bien la validación original obtiene un puntaje general y confiabilidad total y en los análisis exploratorios finales optan por recomendar el uso del puntaje general, se trata de una prueba multidimensional en la que en diversos modelos exploratorios la dimensión de marihuana constituía un área distinta del alcohol y tabaco, entre los cuales hay mayores cargas cruzadas, además de ser el factor con mayor porcentaje de varianza explicada (Carpenter \& Howard, 2009).

En ese sentido, es esperable que las tres sustancias como dimensiones correlacionen alto; ya que miden el mismo constructo, pero también porque la redacción de los ítems es esencialmente igual y cubren las mismas situaciones sociales. Sin embargo, se puede concluir por lo anteriormente mencionado y las diferentes soluciones factoriales de la escala, que es factible medir solo la dimensión de marihuana, teniendo en cuenta las características de la subescala y las limitaciones del estudio en cuanto a la extensión de la batería de instrumentos.

Esta adaptación es relevante para la comunidad científica de habla española y en particular en nuestro país, dado que no se han realizado previas adaptaciones de este instrumento en español y se cuenta con escasas medidas validadas de esta variable. Además, como se ha explicado anteriormente, otros instrumentos están orientados a población adulta o ya consumidora y no hacen tanto énfasis en el aspecto social, que es probablemente el contexto en el que se dé el inicio del consumo en la adolescencia. Estos aspectos hacen de esta subescala una herramienta importante que puede ser usada en investigación, prevención o intervención a nivel comunitario o escolar relacionada al consumo de marihuana.

Dada la importancia de esta variable para el inicio e incremento del consumo, así como para su prevención y tratamiento, es clara la necesidad de contar con un instrumento adaptado y validado que mida la autoeficacia para el rechazo de consumo en nuestro medio para adolescentes. Por ello, el propósito del presente estudio es evaluar las evidencias de las propiedades psicométricas de validez y confiabilidad de la subescala de marihuana de la escala de Autoeficacia para el Rechazo de Consumo de Drogas (DURSE) en escolares de colegios nacionales de Lima.

La ventaja de la escala DURSE para este propósito es que las subescalas para marihuana, alcohol y tabaco tienen la particularidad de tener los mismos ítems, solo cambiando el nombre de la sustancia de una subescala a otra, por lo que los procesos realizados de traducción pueden ser fácilmente usados por otros investigadores para evaluar la autoeficacia para el rechazo de estas sustancias, y los análisis de las propiedades psicométricas pueden brindar un buen indicador de cómo se desempeñan las otras subescalas en un contexto de investigación.

\section{Método}

\section{Participantes}

La muestra estuvo conformada por 1015 estudiantes escolares de tercero a quinto de secundaria de colegios nacionales pertenecientes a Lima Metropolitana. Los colegios fueron seleccionados en base a la cantidad de alumnado y disponibilidad para la aplicación. Los estudiantes fueron 533 hombres y 482 mujeres, con un rango de edad entre 13 y 18 años $(M=15.19 ; D E=1.08)$. La prevalencia de vida de consumo de marihuana en los escolares de la muestra fue de $5.6 \%$. Por otro lado, al $28.4 \%$ de escolares le han ofrecido marihuana 
y aproximadamente la mitad de estas ofertas han sucedido en el mismo año de la aplicación; respecto a ello, el 23.25\% refirió haber dicho que no cuando les ofrecieron marihuana. Asimismo, el 30.04\% de adolescentes manifestaron sentir curiosidad por probar marihuana y el 7\% indicó que probaría marihuana si tuviese la oportunidad.

Inicialmente, se encuestó a 1054 estudiantes, de los cuales 21 fueron eliminados del análisis por no haber llenado en su totalidad la ficha de datos personales y 18 fueron eliminados del análisis por haber dejado por lo menos una respuesta de la escala en blanco.

\section{Instrumentos}

Ficha de datos sociodemográficos. Ficha que fue creada para el estudio, en la cual se les preguntó a los participantes su edad, sexo, año de estudios, prevalencia de vida y consumo anual de marihuana; además de factores asociados al consumo como la curiosidad por probar, intención de consumir si tuviera la oportunidad, si le han ofrecido marihuana y si ha aceptado dicha oferta. Asimismo, la ficha incluyó preguntas sobre el consumo de marihuana por parte de los pares, la disponibilidad de la droga y la percepción de riesgo del consumo de marihuana.

Subescala de marihuana de la escala de Autoeficacia para el Rechazo de Consumo de Drogas (DURSE; Carpenter, 2006). Consta de 8 ítems referidos a diversas situaciones en las que un amigo, adulto o familiar ofrecería marihuana a un adolescente. El participante debe responder en un formato de respuesta tipo Likert de 4 puntos su nivel de seguridad para poder rechazar el ofrecimiento de marihuana si así lo quisiera. Las instrucciones contienen la pregunta: «¿Cuán seguro(a) estás de poder decir 'no', si...», y cada ítem contiene una situación específica (por ejemplo, que un amigo le ofreciera marihuana en su casa o en una fiesta).

Cuestionario de Expectativas de los Efectos de Consumo de Marihuana (MEEQ; Schafer \& Brown, 1991). Adicionalmente, como parte de la investigación general, se aplicó este cuestionario, el cual obtuvo una confiabilidad por coeficiente alpha de Cronbach de .94 para expectativas positivas y .92 para expectativas negativas, así como buenos índices de ajuste en el Análisis Factorial Confirmatorio (CFI = $.91 ;$ TLI $=.91 ;$ RMSEA $=.046 ;$ SRMR $=.049)$ (Otiniano et al., 2017).

La Escala Breve de Búsqueda de Sensaciones (BSSS; Hoyle et al., 2002). Así también, se aplicó esta escala, la cual obtuvo una confiabilidad compuesta de 82 y buenos índices de ajuste en el Análisis Factorial Confirmatorio $(\mathrm{CFI}=.98$; $\mathrm{TLI}=.97$; RMSEA $=.046$; SRMR $=.026$ ) (Romero et al., 2017).

Cuestionario de Monitoreo Parental (PMQ; Kerr \& Stattin, 2000). El cual, en una investigación longitudinal, dos años después, obtiene confiabilidades por alpha de Cronbach de .79 y .84 para el área de control parental, .70 y .72 para el área de solicitud parental, así como .72 y .78 para el área de revelación del adolescente (Kerr et al., 2010).

\section{Procedimiento}

En primer lugar, se realizó la traducción de la subescala haciendo uso del método de traducción directa y traducción reversa. Para la traducción del inglés al español se recurrió a dos profesores de Psicología con un nivel avanzado de inglés, mientras que para la traducción del español al inglés se recurrió a una traductora profesional. Tras una aplicación piloto en un colegio nacional a 83 alumnos, se realizaron cambios a las instrucciones dado que, a pesar de la alta confiabilidad que obtuvo en el piloto, la mayoría de los alumnos respondían marcando la opción más alta. Se hipotetizó que podían estar respondiendo en base a una actitud negativa hacia la marihuana más que en base a contemplar su propia percepción de su capacidad para rechazar las ofertas en situaciones distintas, con diferente dificultad. De esta manera, se intentó reducir la deseabilidad social al enfatizar que son situaciones hipotéticas y de distinto grado de dificultad. Asimismo, se realizaron ligeros cambios al ítem 2 («Un amigo o amiga mayor, 
hermano o hermana te ofrece marihuana en una fiesta y tú no quieres») para dar a entender mejor la idea de que se trataba de amigos mayores o hermanos.

Con respecto a la aplicación, el contacto con los colegios se realizó a través de los directores y, una vez obtenidos los permisos, se explicó el propósito general del estudio en los salones a profesores y alumnos. Se otorgó un asentimiento informado a los alumnos y una carta a los padres explicando el propósito del estudio a fin de cumplir con los estándares éticos en la investigación. En esta carta se les dio la oportunidad a los padres de contactarse con los investigadores y decidir retirar los datos de sus hijos/as de la muestra si lo considerasen necesario. Se contó con un aplicador por cada salón de máximo 30 alumnos. Asimismo, la investigación y sus procedimientos fueron aprobados por el comité de ética de la Pontifica Universidad Católica del Perú, como parte de los procedimientos para ser un proyecto aprobado por la Dirección General de Investigación.

\section{Análisis de datos}

Se realizaron los análisis usando el programa $\mathrm{R}$ versión 4.0.3. En primer lugar, se analizó la normalidad multivariada en base a los test de Mardia (1970) y Henze y Zirkler (1990) usando el paquete MVN (Korkmaz et al., 2014). Luego, para llevar a cabo el Análisis Factorial Confirmatorio mediante el paquete lavaan (Rosseel, 2012), se usó el estimador robusto de Máxima Verosimilitud (MLM) el cual es usado para brindar indicadores ajustados cuando no se cumple el supuesto de normalidad multivariada. Asimismo, por esta razón se reporta el chi-cuadrado con la corrección Satorra-Bentler (Satorra \& Bentler, 2001). Dado que el chi-cuadrado suele ser sensible a tamaños de muestra, se usaron otras medidas de bondad de ajuste como el error cuadrático medio aproximación (RMSEA, por sus siglas en inglés), el residual cuadrático medio estandarizado (SRMR, por sus siglas en inglés), el índice de ajuste comparativo (CFI, por sus siglas en inglés) y el índice de Tucker Lewis (TLI, por sus siglas en inglés). Los valores generalmente aceptables de estos índices son RMSEA < .06, SRMR < .08, y CFI y TLI > .95 (Hu $\&$ Bentler, 1999).

Tomando como referencia los análisis de Carpenter (2006) para evaluar la estructura factorial por sexo, se realizará un análisis de invarianza del modelo factorial confirmatorio según sexo. Para ello, se recurrió nuevamente al paquete lavaan, realizando un análisis multigrupo en el que, en primer lugar, el modelo de línea de base probó si un modelo de factor único se mantuvo en las dos muestras sin imponer restricciones de invarianza. Luego, el modelo métrico abordó la invariancia de las cargas factoriales entre los grupos y, finalmente, en el modelo escalar, las cargas factoriales y las intersecciones se restringieron a ser invariantes entre los grupos.

Respecto de la confiabilidad, dado que se ha encontrado que el coeficiente alpha de Cronbach puede subestimar o sobreestimar la confiabilidad bajo ciertas condiciones, se usó el coeficiente omega de McDonald (Dunn et al., 2013). Este fue calculado mediante el paquete MBESS (Kelley, 2020).

Finalmente, para obtener evidencias de validez de criterio concurrentes se hicieron comparaciones de medias con T de Student con el software SPSSv25, la muestra fue dividida en dos grupos de acuerdo a si habían consumido marihuana alguna vez, tenían curiosidad de probar, probarían si tuviesen la oportunidad y si ha dicho que no cuando le han ofrecido marihuana. Para apreciar la magnitud de estas diferencias se tomó en cuenta el tamaño del efecto haciendo el cálculo de la $d$ de Cohen para muestras de tamaños distintos (Lenhard \& Lenhard, 2016) usando los criterios de interpretación de Cohen (1992). 


\section{Resultados}

A continuación, se presentan los resultados de las evidencias de validez mediante análisis factorial y la confiabilidad de la subescala. En primer lugar, para realizar el Análisis Factorial Confirmatorio (AFC), se llevó a cabo previamente un análisis de normalidad multivariada para confirmar este supuesto. Los coeficientes de asimetría (20.86) y curtosis (256.45) multivariada de Mardia (1970) señalan que los datos no presentan normalidad multivariada. Adicionalmente, el test de Henze-Zirkler de normalidad multivariada (Henze \& Zirkler, 1990), el cual resultó significativo con un coeficiente de 279.84, apuntó en la misma dirección. En relación con este hallazgo, se encuentra también que los estudiantes tienden a marcar con alta autoeficacia en todos los ítems, lo cual lleva a estas desviaciones de la normalidad.

Es por ello, justamente, que se usó el estimador MLM, el cual brinda valores robustos que se ven menos afectados por la falta de normalidad multivariada. Este AFC muestra que el modelo unifactorial propuesto $\left(\chi^{2}=85.073 ; d f=20 ; p=\right.$ $\left..000 ; \mathrm{S}-\mathrm{B} \chi^{2}=4.227\right)$ tiene buenos indicadores de bondad de ajuste (CFI = .990; TLI = .986; RMSEA $=.057[.051-.063]$; SRMR $=.016$ ), a pesar de tener un $\chi^{2}$ significativo, lo cual suele suceder en muestras superiores a $N=400$ y cuando hay alta correlación entre las variables, por lo que se le considera una medida muy liberal de ajuste (Kenny, 2020). Sin embargo, el resto de los indicadores comúnmente reportados en análisis confirmatorios coinciden en un buen nivel de ajuste. Por otro lado, la confiabilidad mediante el coeficiente omega de McDonald es de .98 I.C. [.973 - .981] lo cual constituye un nivel alto de confiabilidad.

Lo encontrado en relación a la distribución de los ítems y el alto coeficiente de confiabilidad podrían indicar la posibilidad de que los ítems estén midiendo información redundante, a pesar de las diversas situaciones sociales que plantean. Esto podría ser un problema que afecte el AFC en el caso de haber usado la prueba en su totalidad, ya que los factores de cada sustancia podrían tener una alta covarianza al indicar multicolinealidad, lo cual no se daría en modelos unifactoriales (Muthen, 2005).

A continuación, se puede apreciar en la Figura 1 el modelo de ecuaciones estructurales del análisis factorial confirmatorio de la escala.

Siguiendo los análisis de Carpenter (2006) en relación con las diferencias por sexo se encontró que las mujeres $(M=25.68 ; D E=8.91)$ tienen puntajes mayores que los hombres $(M=26.94, D E=7.991)$. Estas diferencias fueron significativas $(t=-2.372$; $p=.018 ; d$ de Cohen $=.149$ ), aunque con un tamaño del efecto pequeño (Cohen, 1992). La distribución de los puntajes en hombres y mujeres es muy similar.

Pese a no encontrar estas diferencias, se llevó a cabo un análisis de invarianza dado que en la validación original se evaluó el modelo factorial exploratorio por sexo, lo correspondiente en AFC sería al análisis de invarianza en el cual se compara el ajuste entre cada grupo según sexo. En un primer paso se prueba la invarianza métrica, en la cual se encontró que el cambio en chi-cuadrado no fue significativo $\left(\Delta \chi^{2}[7]=12.334 ; p=.09\right)$. Esto indica que no existen diferencias importantes en las cargas factoriales entre ambos grupos y que para ambos el modelo unifactorial es el mismo y posee la misma estructura. Por otro lado, al analizar la invarianza escalar se encontró que la diferencia entre chi-cuadrado fue significativa ( $\Delta \chi^{2}[14]$ $=25.588 ; p=.03$ ). Ello indica que no hay equivalencia escalar entre los grupos; es decir que hay diferencias entre los interceptos de ambos grupos. Esto evidencia una respuesta diferencial según sexo, por lo que sus puntajes no pueden ser comparados en relación con la variable latente. En la Figura 2 se puede apreciar los modelos por grupo. 
Figura 1

AFC de la subescala de marihuana de la escala DURSE

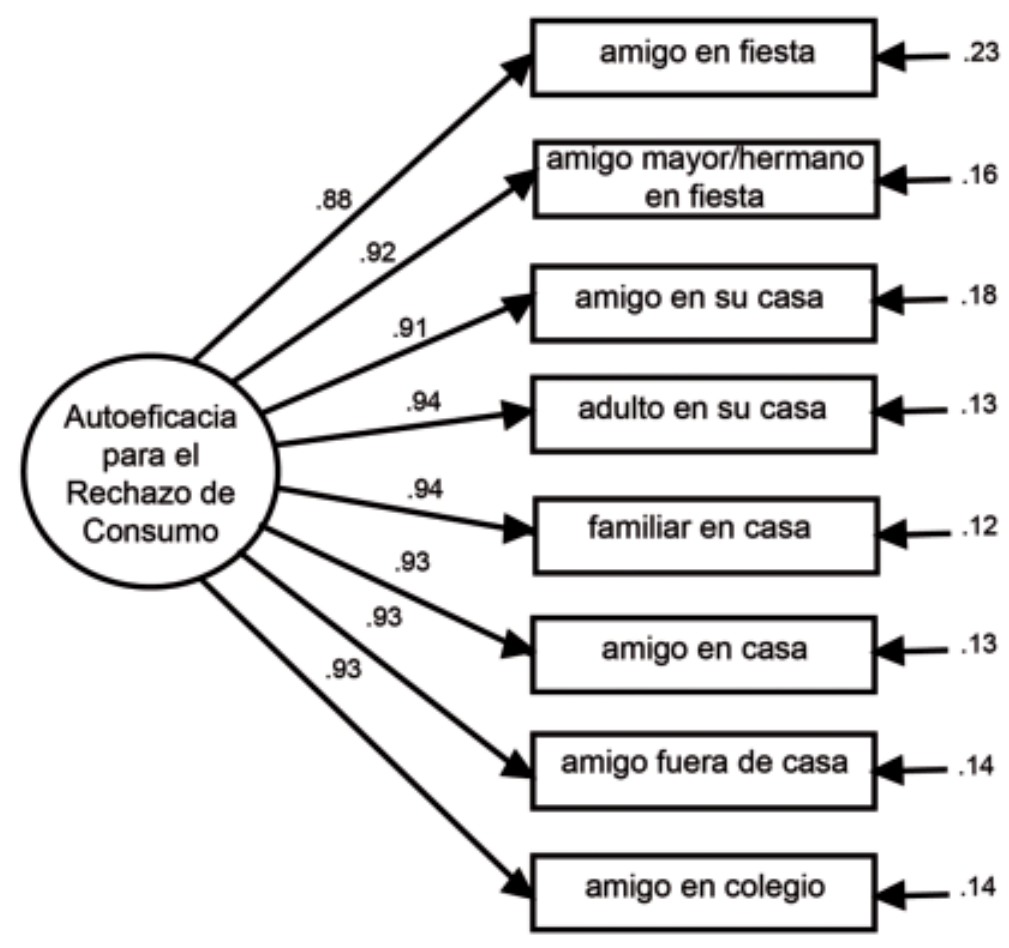

Figura 2

AFC de la subescala de marihuana de la escala DURSE por sexo del participante

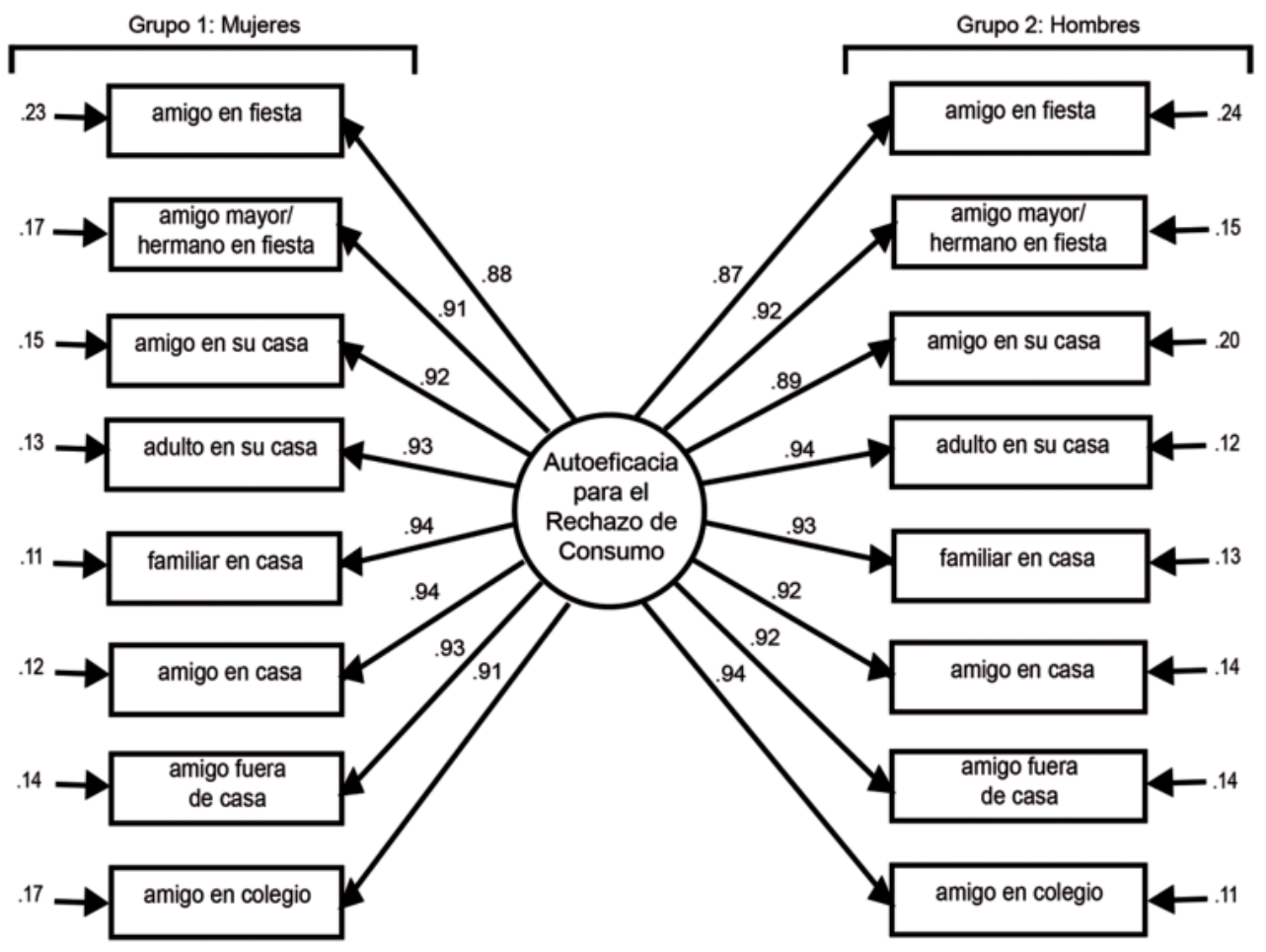


Por otro lado, se obtuvieron evidencias de validez de criterio usando la subescala de marihuana como variable dependiente para comparar medias. Se encontró que la autoeficacia fue significativamente menor con un tamaño del efecto medio en el grupo de adolescentes que no había consumido marihuana y en el grupo que probaría si tuviese la oportunidad, mientras que no hubo diferencias significativas entre aquellos que tenían o no curiosidad de probar marihuana (Tabla 1). Finalmente, la autoeficacia fue significativamente mayor con un tamaño del efecto mediano en aquellos a los que les habían ofrecido marihuana y habían rechazado dicha oferta.

Tabla 1

Diferencia de medias de puntaje en la subescala de marihuana de la escala DURSE según variables relativas al consumo

\begin{tabular}{|c|c|c|c|c|c|c|c|c|}
\hline \multirow{2}{*}{ Variable } & \multicolumn{2}{|c|}{ Sí } & \multicolumn{2}{|c|}{ No } & \multirow{2}{*}{$t$} & \multirow{2}{*}{$g l$} & \multirow{2}{*}{$p$} & \multirow{2}{*}{$\begin{array}{c}d \\
\text { de Cohen }\end{array}$} \\
\hline & $M$ & $D E$ & $M$ & $D E$ & & & & \\
\hline $\begin{array}{l}\text { Ha consumido } \\
\text { marihuana alguna } \\
\text { vez }\end{array}$ & 22.04 & 8.093 & 26.56 & 8.454 & -3.935 & 999.00 & .000 & .536 \\
\hline $\begin{array}{l}\text { Curiosidad de } \\
\text { probar* }\end{array}$ & 25.62 & 7.673 & 26.63 & 8.780 & -1.822 & 655.49 & .069 & .119 \\
\hline $\begin{array}{l}\text { Probaría si tuviese } \\
\text { la oportunidad }\end{array}$ & 20.99 & 7.811 & 26.73 & 8.383 & -5.595 & 1003.00 & .000 & .688 \\
\hline $\begin{array}{l}\text { Ha dicho que no } \\
\text { cuando le } \\
\text { ofrecieron** }\end{array}$ & 26.49 & 7.499 & 22.58 & 8.377 & 3.367 & 287.00 & .001 & .513 \\
\hline
\end{tabular}

Nota: *no se asumen varianzas iguales; ${ }^{* *} n=289$, ya que se excluyó a quienes no les ofrecieron.

\section{Discusión}

En primer lugar, se observa que los resultados encontrados están en línea con los estudios de Carpenter y Howard (2009), quienes también hallan altas cargas factoriales, niveles similares de confiabilidad y una tendencia similar a responder de manera positiva, señalando una alta autoeficacia para el rechazo de consumo de marihuana. Esta tendencia positiva ha sido reportada en estudios previos en escolares de Estados Unidos de menor edad en promedio a la de los escolares de esta investigación con el mismo instrumento, teniendo una tendencia positiva más alta a la subescala de marihuana (Carpenter \& Howard, 2009). Estas tendencias han sido reportadas en otras escalas de autoeficacia para el rechazo, como la Wake Forest Drug Refusal Skill
Scale y más aun con la Botvin Refusal Skills Scale (Carpenter, 2006); mas no en otras como el CRSEQ, el DASES y el DTCQ, que son más usadas en adultos y población que consume drogas. Esto puede deberse a la deseabilidad social, ya que los adolescentes probablemente consideran el rechazo de drogas como un comportamiento socialmente aceptable, especialmente en el contexto del colegio, e investigaciones anteriores han encontrado una asociación pequeña entre la escala DURSE y la deseabilidad social (Carpenter \& Howard, 2009). En ese sentido, la investigación trató de limitar el impacto de la deseabilidad en las modificaciones a las instrucciones desde el piloto, pese a ello, una limitación del estudio es no haber contado con una medida o indicador de la deseabilidad social que pudiera haber servido parar comprobar esta hipótesis. 
Asimismo, esto puede ser producto de la falta de experiencia y familiaridad con las drogas, lo cual podría llevar a una sobreestimación de su autoeficacia (Carpenter \& Howard, 2009), puesto que muchas veces la medición de la autoeficacia no está dirigida a medir la confianza de la persona en realizar la conducta ocasionalmente, sino de manera usual en diversas situaciones (Bandura, 2006). En ese sentido, dado que las situaciones en las que les ofrecen marihuana son poco usuales y solo han sido experimentadas por el $28 \%$ de la muestra, es posible que los alumnos tuvieran pocos elementos para hacer un juicio apropiado de su autoeficacia. Sin embargo, la importancia de la experiencia previa podría ser relativa dado que en la escala CRSEQ orientada a población clínica adulta también se han detectado problemas de asimetría en los ítems (Young et al., 2012). Cabe resaltar que de este porcentaje al que le ofrecieron marihuana solo el $22.45 \%$ aceptó, lo cual podría deberse a una verdadera alta autoeficacia para el rechazo o, nuevamente, a la deseabilidad social.

En ese sentido, durante la aplicación de los instrumentos, se pudo apreciar de forma cualitativa que varios estudiantes manifestaron, al responder la prueba, que no deseaban consumir marihuana, por lo que sería factible que algunos puedan haber respondido de manera general basándose en su propia tendencia a rechazar el consumo sin considerar de manera individual las situaciones de diversa dificultad que presenta la subescala.

A pesar de estas dificultades, la subescala de marihuana de la escala DURSE muestra propiedades adecuadas de validez interna, ajustándose los datos a la estructura interna propuesta por Carpenter y Howard (2009) de acuerdo con los criterios de Hu y Bentler (1999). En ese sentido, evaluar las propiedades de validez mediante un Análisis Factorial Confirmatorio supone una mejora con relación a la investigación original, la cual es posible en parte por contar con una muestra más amplia. Asimismo, la escala logra una alta consistencia interna (Nunnally \& Bernstein, 1995) similar a la hallada en estudios anteriores, aunque esta vez medida con el coeficiente omega, que supera cuestionamientos hechos a medidas comúnmente usadas como el alfa de Cronbach (Dunn et al., 2013).

Sin embargo, se deben tomar en cuenta estos aspectos en futuras investigaciones que adapten la escala completa, ya que la distribución de respuesta podría indicar que hay ítems que miden información redundante o que los adolescentes no llegan a distinguir tanta diferencia entre algunas situaciones sociales planteadas. Esto podría llevar, por un lado, a estudios piloto más exhaustivos para replantear la redacción de los ítems para destacar estas diferencias o a eliminar ítems que midan situaciones igualmente difíciles para los adolescentes a través de todas las sustancias.

En relación con las diferencias por sexo del participante, si bien no se encontraron diferencias en los niveles de autoeficacia para el rechazo, el análisis de invarianza factorial supone un aporte a los análisis realizados en la validación inicial de la prueba, encontrándose que la estructura interna del constructo no varía para hombres y mujeres. Sin embargo, al no hallarse invarianza escalar, esto indica que algunos ítems pueden estar llevando a que mujeres u hombres puntúen más alto o bajo. Esto puede deberse a las diferentes percepciones y expectativas que tienen hombres y mujeres sobre estas situaciones, sobre cuáles son más realistas, y en cuáles tendrían mayor disposición a rechazar la oferta (Carpenter, 2006).

Por otro lado, la prueba ha logrado mostrar evidencias de validez de criterio al encontrarse niveles más bajos de autoeficacia para el rechazo en quienes consumieron alguna vez y en quienes probarían si tuvieran la oportunidad, lo cual se alinea con investigaciones previas que encuentran relación entre la autoeficacia, el consumo y la intención de consumo (D’Amico et al., 2020; Lopez-Quintero \& Neumark, 2015; Morell-Gomis et al., 2019); dicha relación con la intención también es hallada por las autoras originales (Carpenter \& Howard, 2009). Asimismo, se muestra un mayor nivel de autoeficacia entre 
aquellos que reportaron haber rechazado ofertas de consumo, lo cual demuestra que la escala es capaz de distinguir a aquellos que muestran dicha conducta. No se encontraron diferencias entre aquellos que tenían o no curiosidad de probar, en este aspecto no había una hipótesis clara al no haber algún hallazgo relevante en la literatura al respecto.

Estos aspectos relacionados de la curiosidad, intención, consumo previo y rechazo de ofertas pueden ser mejor entendidos en teorías unificadas como la Teoría de la Acción Planeada (Guzmán et al., 2012) según la cual las actitudes, normas sociales, control percibido y real sobre el comportamiento son predictores de la intención, la cual a su vez predice la conducta de consumo.

En suma, estas propiedades psicométricas evidencian que la subescala es adecuada para su uso en investigaciones u otros propósitos y es capaz de contribuir a la explicación del inicio de consumo de marihuana en adolescentes escolares. Sin embargo, se debe tener en cuenta las limitaciones mencionadas anteriormente en relación con la distribución de los puntajes y la posible influencia de deseabilidad social. Otras limitaciones generales de esta investigación refieren a que, si bien la muestra tiene un tamaño representativo en relación con la población escolar de Lima, los colegios no fueron seleccionados aleatoriamente; por lo que podría haber ciertas limitaciones para extrapolar estos hallazgos a la población y al país en su totalidad.

En posteriores investigaciones sería importante distinguir la medición de la autoeficacia para el rechazo de las actitudes negativas hacia el consumo de marihuana. Si bien estas son variables relacionadas, sería importante, para establecer la validez discriminante de la escala, que esta pueda distinguir la capacidad percibida para rechazar las ofertas de las actitudes negativas previas hacia la sustancia. Asimismo, sería menester esclarecer el rol de la deseabilidad social de acuerdo con lo discutido previamente y ahondar en las diferencias por sexo en cuanto a situaciones de oferta de consumo y la percepción diferenciada de las mismas o de su respuesta social en cuanto a aceptar o rechazar dichas ofertas. Ello podría explicar las causas de la invarianza a nivel métrico y proveer de mejores conocimientos para la medición de la autoeficacia para el rechazo según sexo.

Por otro lado, dado que se realizaron adecuados procedimientos de traducción, y la subescala presenta adecuadas propiedades psicométricas, futuros investigadores pueden disponer de la traducción realizada para adaptar rápidamente los ítems de las demás subescalas. Esto es de particular utilidad en nuestro país dada la escasez de instrumentos validados no solo en español, sino también validados en el Perú. En ese sentido, la escala DURSE se destaca sobre otras escalas similares usadas en la investigación, al medir diversas situaciones de presión social, ya que hallazgos previos reportaron que algunos encuestados entendían preguntas generales como «si te la ofreciera un amigo» o «un mejor amigo» de una manera particular, dado que podían pensar que un verdadero o buen amigo no les invitaría a probar sustancias. Asimismo, esta escala incorpora situaciones en casa y con familiares, lo cual permite evaluar factores familiares en el inicio del consumo (Carpenter \& Howard, 2009). Esto evidencia la utilidad de esta subescala en investigación de factores predictores del consumo, así como para su uso en intervenciones o acciones de prevención.

$\mathrm{Al}$ adaptar las dos subescalas restantes de la escala DURSE en posteriores investigaciones, sería importante examinar las variaciones en los patrones de respuesta para las distintas sustancias. Además, al investigar en poblaciones vulnerables (en tratamiento o con conductas adictivas) sería relevante analizar la relación entre la autoeficacia para el rechazo de ofertas de consumo y la autoeficacia para no consumir en general o para la abstinencia, dada la importancia que pueden tener ambos aspectos en la prevención y tratamiento. En suma, esta investigación aporta al cuerpo de instrumentos psicológicos validados en 
español para la medición de variables relacionadas al consumo de marihuana en adolescentes.

\section{Conflicto de intereses}

Los autores no tienen conflictos de intereses morales, económicos, laborales o de investigaciones relacionadas al presente estudio.

\section{Responsabilidad ética}

El presente estudio no involucró experimentación humana o animal, se siguieron los procedimientos de asentimiento informado de los adolescentes y consentimiento de los padres. Con la finalidad de asegurar la confidencialidad de los datos, se separó los consentimientos de los protocolos eliminando toda forma de identificación. Asimismo, el estudio no detalla ningún dato individual de las respuestas de los estudiantes o ningún dato que permita su identificación. El estudio fue revisado y aprobado por el Comité de Ética para la Investigación con Seres Humanos y Animales (CEIsha) de la PUCP.

\section{Contribución de autoría}

DPOC: diseño del estudio, análisis de los datos, elaboración de la discusión y revisión final del manuscrito.

CCPA: concepción, diseño y supervisión del estudio, revisión de la discusión y conclusiones.

FOC: diseño del estudio, revisión del análisis de datos, revisión final del manuscrito.

SMRS: diseño del estudio, supervisión de los procedimientos metodológicos y análisis de invarianza.

\section{Agradecimiento}

Este trabajo fue financiado por la Dirección de Gestión de la Investigación de la Pontificia Universidad Católica del Perú a través del financiamiento DGI-2014-0032.

\section{Referencias}

Bandura, A. (1986). Social Foundations of Thought and Action: A Social Cognitive Theory. Prentice Hall.

Bandura, A. (2001). Social Cognitive Theory: An Agentic Perspective. Annual Review of Psychology, 52(1), 126. https://doi.org/10.1146/annurev.psych.52.1.1

Bandura, A. (2006). Guide for Constructing Self-Efficacy Scales. En F. Pajares \& T. Urdan (Eds.), Self-Efficacy Beliefs of Adolescents (Vol. 5, pp. 307-337). Information Age Publishing.

Carpenter, C. (2006). Development of a Scale to Measure Adolescent's Drug Use Resistance Self-Efficacy [tesis doctoral, Universidad de Maryland]. Repositorio: http:/ /hdl.handle.net/1903/3498

Carpenter, C., \& Howard, D. (2009). Development of a Drug Use Resistance Self-Efficacy (DURSE) Scale. American Journal of Health Behavior, 33(2), 147-157. https:// doi.org/10.5993/AJHB.33.2.4

Centro de Información y Educación para la Prevención del Abuso de Drogas [CEDRO]. (2017). Epidemiología de Drogas en Población Urbana Peruana: Encuesta en Hogares. http://repositorio.cedro.org.pe/bitstream/ CEDRO/361/3/CEDRO.Estudio\%20EPI. 2017.pdf

Centro de Información y Educación para la Prevención del Abuso de Drogas [CEDRO]. (2018). El Problema de las Drogas en el Perú. http://www.repositorio.cedro.org.pe/ bitstream/CEDRO/378/1/CEDRO.Problema\%20de\% 20las\%20drogas.2018.pdf

Choi, H., Krieger, J., \& Hecht, M. (2013). Reconceptualizing Efficacy in Substance Use Prevention Research: Refusal Response Efficacy and Drug Resistance SelfEfficacy in Adolescent Substance Use. Health Communication, 28(1), 40-52. https://doi.org/10.1080/ 10410236.2012.720245

Chung, T., \& Maisto, S. (2016). Time-Varying Associations between Confidence and Motivation to Abstain from Marijuana During Treatment Among Adolescents. Addictive Behaviors, 57, 62-68. https://doi.org/10.1016/ j.addbeh.2016.02.015

Cohen, J. (1992). A Power Primer. Psychological Bulletin, 112(1), 155-159. https://doi.org/10.1037/00332909.112.1.155 
Comisión Nacional para el Desarrollo y Vida sin Drogas [DEVIDA]. (2014a). Consumo problemático de marihuana en la población escolar de secundaria (Validación de la Escala CAST Cannabis Abuse Screening Test). https://www.devida.gob.pe/ documents/20182/97343/consumo-problematicomarihuana-Peru.pdf/b90f52be-8950-4678-afedcc97f8c7b477

Comisión Nacional para el Desarrollo y Vida sin Drogas [DEVIDA]. (2014b). Manual para la prevención del consumo de drogas. Proyecto de Descentralización de Políticas de Drogas en los Países Andinos PERÚ. https://www.devida.gob.pe/documents/20182/97343/ Manual-para-la-prevenci\%C3\%B3n-del-consumo-dedrogas.pdf/6bbbfe1b-b23f-4796-8c24-5c73583be8ff

D’Amico, E., Rodriguez, A., Tucker, J., Dunbar, M., Pedersen, E., Shih, R., Davis, J., \& Seelam, R. (2020). Early and Late Adolescent Factors that Predict Co-Use of Cannabis with Alcohol and Tobacco in Young Adulthood. Prevention Science, 21, 530-544. https://doi.org/10.1007/ s11121-020-01086-7

Dunn, T., Baguley, T., \& Brunsden, V. (2013). From Alpha to Omega: A Practical Solution to the Pervasive Problem of Internal Consistency Estimation. British Journal of Psychology, 105(3), 399-412. https://doi.org/10.1111/ bjop.12046

Etchemendi, M. C., Rodríguez, M., \& Valenzuela, R. (2019). Adaptación y validación de la escala de autoeficiencia para la evitación de drogas (DASESThe Drug Avoidance Self-Efficacy Scale) [tesis de pregrado, Universidad Nacional de Mar del Plata]. Repositorio RPsico. http://rpsico.mdp.edu.ar/handle/ 123456789/1151

Gullo, M., Matveeva, M., Feeney, G., Young, R., \& Connor, J. (2017). Social Cognitive Predictors of Treatment Outcome in Cannabis Dependence. Drug and Alcohol Dependence, 170, 74-81. https://doi.org/10.1016/j.druga lcdep.2016.10.030

Guzmán, F., Llamas, M., Rodríguez, L., \& Alonso, M. (2012). Norma subjetiva, intención y consumo de marihuana en jóvenes universitarios de México. Ciencia y Enfermería, 18(1), 57-66. http://dx.doi.org/ 10.4067/S0717-95532012000100006
Henze, N., \& Zirkler, B. (1990). A Class of Invariant Consistent Tests for Multivariate Normality. Communications in Statistics - Theory and Methods, 19(10), 3595-3617. https://doi.org/10.1080/0361092900 8830400

Hoyle, R., Stepenson, M., Palmgreen, P., Pugzles, E., \& Donohew, L. (2002). Reliability and Validity of a Brief Measure of Sensation Seeking. Personality and Individual Differences, 32(3), 401-414. https://doi.org/ 10.1016/S0191-8869(01)00032-0

Hu, L., \& Bentler, P. M. (1999). Cutoff Criteria for Fit Indexes in Covariance Structure Analysis: Conventional Criteria versus New Alternatives. Structural Equation Modeling: A Multidisciplinary Journal, 6(1), 1-55. https://doi.org/10.1080/10705519909540118

Kelley, K. (2020). MBESS: The MBESS R Package. $R$ package version 4.8.0. https:/CRAN.R-project.org/ package $=$ MBESS

Kenny, D. (2020, 5 de junio). Measuring Model Fit. http:// www.davidakenny.net/cm/fit.htm

Kerr, M., \& Stattin, H. (2000). What Parents Know, How They Know it, and Several Forms of Adolescent Adjustment: Further Support for a Reinterpretation of Monitoring. Developmental Psychology, 36(3), 366380. https://doi.org/10.1037/0012-1649.36.3.366

Kerr, M., Stattin, H., \& Burk, W. (2010). A Reinterpretation of Parental Monitoring in Longitudinal Perspective, Journal of Research on Adolescence, 20(1), 39-64. https://doi.org/10.1111/j.1532-7795.2009.00623.x

Korkmaz, S., Goksuluk, D., \& Zararsiz, G. (2014). MVN: An $\mathrm{R}$ Package for Assessing Multivariate Normality. The R Journal, 6(2), 151-162.

Lenhard, W., \& Lenhard, A. (2016). Calculation of Effect Sizes. https://www.psychometrica.de/effect_size.html

Lopez-Quintero, C., \& Neumark, Y. (2015). Prevalence and Determinants of Resistance to Use Drugs Among Adolescents Who had an Opportunity to use drugs. Drug and Alcohol Dependencies, 149(1), 55-62. https://dx.doi.org/10.1016\%2Fj.drugalcdep.2015.01.015

Naciones Unidas. (2019). World Drug Report 2019. National Health Promotion Associates. https://wdr.unodc.org/ wdr2019/index.html 
National Health Promotion Associates. (2017). Botvin Life Skills ${ }^{\circledR}$ Training. Planning Workbook. A Guide for Implementing a LifeSkills Training Program. https:// www.lifeskillstraining.com/wp-content/uploads/2017/ 10/LST-Planning-Workbook.pdf

Nunnally, J., \& Bernstein, I. H. (1995). Teoría psicométrica. McGraw-Hill

Mardia, K. V. (1970). Measures of Multivariate Skewness and Kurtosis with Applications. Biometrika, 57(3), 519530. https://doi.org/10.2307/2334770

Martin, G., Wilkinson, D., \& Poulos, C. (1995). The Drug Avoidance Self-Efficacy Scale. Journal of Substance Abuse, 7(2), 151-163. https://doi.org/10.1016/08993289(95)90001-2

Morell-Gomis, R., Moriano, J., Laguía, A., Dias, P., \& Lloret, D. (2019). Adolescents Cannabis Use Intention: Validating a Theory of Planned Behavior Questionnaire in Four European Countries. Journal of Substance Use, 24(1), 66-72. https://doi.org/10.1080/14659891.2018.15 10050

Muthen, B., (2005). Multicolinearity in Confirmatory Factor Analysis. Foro de Mplus. http://www.statmo del.com/discussion/messages/9/905.html?1496883730

Organización de los Estados Americanos [OEA]. (2019). Informe sobre el consumo de drogas en las Américas 2019. OEA. http://www.cicad.oas.org/main/pubs/Infor me\%20sobre\%20el\%20consumo\%20de\% 20drogas \%20en\% 20las\%20Am\%C3\%A9ricas\%202019.pdf

Otiniano, F., Romero, S., Olivo, D., \& Chau, C. (2017). Adaptación y propiedades psicométricas del Marijuana Effect Expectancy Questionnaire (MEEQ) en adolescentes escolares peruanos. Revista Mexicana de Psicología, 34(2), 125-133.

Papinczak, Z., Connor, J., Harnett, P., \& Gullo, M. (2018). A Biosocial Cognitive Model of Cannabis Use in Emerging Adulthood. Addictive Behaviors, 76, 229235. https://doi.org/10.1016/j.addbeh.2017.08.011

Pearson, M. R., Bravo, A. J., \& Protective Strategies Study Team. (2019). Marijuana Protective Behavioral Strategies and Marijuana Refusal Self-Efficacy: Independent and Interactive Effects on MarijuanaRelated Outcomes. Psychology of Addictive Behaviors, 33(4), 412-419. https://doi.org/10.1037/adb0000445
Ramo, D., Myers, M., \& Brown, S. (2009). Psychometric Properties of a Revised Form of the Drug-Taking Confidence Questionnaire for Use with Adolescents. Journal of Child \& Adolescent Substance Abuse, 18(1), 24-42. https://doi.org/10.1080/15470650802541061

Real Prevention. (2019). Keepin' it Real. https://realprevention.com/keepin-it-real/

Romero, S., Olivo, D., Chau, C., \& Otiniano, F. (2017). Psychometric Properties of the Brief Sensation Seeking Scale in Peruvian Teenagers. Psicothema, 29(1), 133138. https://doi.org/10.7334/psicothema2016.144

Rosseel, Y. (2012). lavaan: An R Package for Structural Equation Modeling. Journal of Statistical Software, 48(2), 1-36. https://doi.org/10.18637/jss.v048.i02

Satorra, A., \& Bentler, P. M. (2001). A Scaled Difference Chi-Square Test Statistic for Moment Structure Analysis. Psychometrika, 66, 507-514.

Schafer, J., \& Brown, S. (1991). Marijuana and Cocaine Effect Expectancies and Drug Use Patterns. Journal of Consulting and Clinical Psychology, 59(4), 558-565. https://doi.org/10.1037/0022-006X.59.4.558

Sheykhnezhad, F., \& Seyedfatemi, N. (2019). Effect of group education on self-efficacy and craving tendencies in drug abusers in 5th Azar Drug Abuse Treatment Center of Gorgan. Cogent Psychology, 6(1), 1-16. https://doi. org/10.1080/23311908.2019.1587818

Shrier, L., Sarda, V., Jonestrask, C., \& Kim, S. (2018). Momentary Factors During Marijuana Use as Predictors of Lapse During Attempted Abstinence in Young Adults. Addictive Behaviors, 83, 167-174. https://doi.org/10.1016/j.addbeh.2017.12.032

Sklar, S., Annis, H., \& Turner, N. (1997). Development and Validation of the Drug-Taking Confidence Questionnaire: A Measure of Coping Self-Efficacy. Addictive Behaviors, 22(5), 655-670. https://doi.org/ 10.1016/S0306-4603(97)00006-3

University of Southern California Institute for Prevention Research. (2019). TND Project Towards No Abuse. Preview. Consultado en febrero de 2020. https:// tnd.usc.edu/?page_id=41

U.S. Department of Health and Human Services. (2016). Facing Addiction in America: The Surgeon General's 
Report on Alcohol, Drugs, and Health. HHS. https:// addiction.surgeongeneral.gov/sites/default/files/ surgeon-generals-report.pdf

Vigo, A. (2003). Adaptación psicométrica del cuestionario de confianza ante situaciones de riesgo para consumir en pacientes adictos [tesis de pregrado, Universidad Nacional Federico Villareal]. Repositorio Institucional UNFV. http://repositorio.unfv.edu.pe/handle/UNFV/948

Walton, M., Bohnert, K., Resko, S., Barry, K., Chermack, S., Zucker, R., Zimmerman, M., Booth, B., \& Blow, F. (2013). Computer and Therapist Based Brief Interventions Among Cannabis-Using Adolescents Presenting to Primary Care: One-Year Outcomes. Drug and Alcohol Dependence, 132(3), 646-653. https://doi.org/10.1016/ j.drugalcdep.2013.04.020

Young, R., Gullo, M., Feeney, G., \& Connor, J. (2012). Development and Validation of the Cannabis Refusal Self-Efficacy Questionnaire (CRSEQ) in Adult Cannabis
Users in Treatment. Drug and Alcohol Dependence, 125(3), 244-251. https://doi.org/10.1016/j.drugalcdep. 2012.02.018

Zavaleta, A., Castro de la Mata, R., Maldonado, V., \& Romero, E. (2006). Características y opiniones sobre drogas en escolares de cuarto y quinto de secundaria. Encuesta Flash. CEDRO.

Zullig, K., \& Valois, R. (2017). Perceptions of Harm from Substance Use and Social Self-Efficacy Among Early Adolescents. Journal of Drug Education, 46(3-4), 96112. https://doi.org/10.1177/0047237917735043

Zvolensky, M., Paulus, D., Garey, L., Manning, K., Hogan, J., Buckner, J., Rogers, A., \& McHugh, K. (2018). Perceived Barriers for Cannabis Cessation: Relations to Cannabis Use Problems, Withdrawal Symptoms, and Self-Efficacy for Quitting. Addictive Behaviors, 76, 4551. https://doi.org/10.1016/j.addbeh.2017.07.011 


\section{David Pascual Olivo Chang}

Pontificia Universidad Católica del Perú, Perú.

Licenciado en Psicología, especializaciones en terapia cognitiva y análisis de datos, analista en Psicología del Observatorio del Delito y la Criminalidad de la PNP, asistente de investigación en proyectos del grupo de investigación Psicología de las Adicciones y Comportamientos de Riesgo (ACR) de la PUCP.

ORCID: https://orcid.org/0000-0001-7384-7809

Autor corresponsal: d.olivo@pucp.pe, david.olivo.chang@gmail.com

Cecilia Chau Pérez-Aranibar

Pontificia Universidad Católica del Perú, Perú.

Doctora en Psicología por la Universidad KU Leuven, Bélgica, docente principal del Departamento de Psicología de la PUCP, y coordinadora del grupo de investigación Psicología de las Adicciones y Comportamientos de Riesgo (ACR) de la PUCP.

ORCID: https://orcid.org/0000-0002-2631-0301

cchau@pucp.pe

Fiorella Otiniano Campos

Pontificia Universidad Católica del Perú, Perú.

Licenciada en Psicología, coordinadora de investigación y desarrollo de la Dirección de Formación Extraacadémica de la Universidad del Pacífico, y miembro del grupo de investigación Psicología de las Adicciones y Comportamientos de Riesgo (ACR) de la PUCP.

ORCID: https://orcid.org/0000-0001-6061-5427

f.otiniano@pucp.pe

\section{Silvana Melissa Romero Saletti}

Pontificia Universidad Católica del Perú, Perú.

Magíster en Metodología de las Ciencias del Comportamiento y la Salud por la UNED, España, magíster en Estudios Teóricos en Psicoanálisis de la PUCP, Perú, becaria de Doctorado en Psicología y Ciencias de la Educación, Université Catholique de Louvain, Bélgica, y profesora a tiempo parcial por asignatura (TPA) en la Pontificia Universidad Católica del Perú.

ORCID: https://orcid.org/0000-0001-7252-8240

silvana.romero@pucp.edu.pe 